\title{
Implementation of the electronic sector of the satellite camera and image contrast enhancement
}

\author{
Majid Zarie, Jafar Khalilpour, Farhad Sadeghi Almaloo
}

Electrical Engineering Department, Khatam al-anbia (pbuh) University, Iran

\begin{tabular}{l}
\hline \hline Article Info \\
\hline Article history: \\
Received Feb 12, 2020 \\
Revised Apr 16, 2020 \\
Accepted Apr 30, 2020 \\
\hline
\end{tabular}

\section{Keywords:}

Contrast enhancement

Detector sector

Entropy

Histogram equalization

Processor sector

Satellite camera

\begin{abstract}
The purpose of this paper is to design and implement the electronic sector of satellite camera on the basis of systematic calculations as well as presenting the general and detailed block diagram. The main parts of the designed camera consist of four units named "optics, detector, processors, and memory" that can work in one of three modes: real-time, storage and storagesend. Verification of the simulation and practical results are shown completely by receiving images. According to the conditions of the satellite imaging, in most cases, there is a need to improve the image quality. A very important feature in the satellite images is contrast enhancement. In the following, a powerful contrast enhancement algorithm is proposed based on histogram equalization method called the "entropy-based triple dynamic clipped histogram equalization" (ETDCHE). One of the strengths of this paper is using images with diverse variations in brightness. Such that by producing clear images and by preserving maximum details this overcomes the adverse lighting conditions and leads to natural enhancement of the output images. Also, the performance assessment of the proposed method in terms of the average information content reflects the considerable superiority of the proposed algorithm compared to the previously presented methods based on histogram equalization.
\end{abstract}

Copyright $@ 2020$ Institute of Advanced Engineering and Science. All rights reserved.

Corresponding Author:

Majid Zarie,

Electrical Engineering Department,

Khatam al-anbia (pbuh) University, Tehran, Iran.

Email: majidzarie@yahoo.com

\section{INTRODUCTION}

Satellite images are used in a wide range of applications such as different scientific studies like geology, astronomy, geographical information systems and observing objects on the ground. In this paper the aim is design and fabrication of satellite imaging boards with an emphasize on improving image quality. The considered design and fabrication process for the imaging board is as investigated in the first step of block diagrams [1-3]. Each of these block diagrams is designed for a different purpose and has specific duties and characteristics. Then, according to the raised demands in design and systematic calculation a block diagram based on main units of optic, detector, processor and memory are proposed. The general process is as follows: the optical section of the camera after collecting light from the ground and aligning finally develops a real image of the scene on the detector. The received light is created as an electrical charge in detector pixels that is proportional to the value of existing energy in incident light. The received analog output from the detector enters the processing section after a series of sampling, amplifying and digitalization. The processing section as the main core of data management and processing is closely related to detector section, memory, and central computer unit so that finally the output image would be prepared for sending or storage.

For imaging three different kinds of the whiskbroom, pushbroom, and snapshot can be used that the dominant choice in most of the imaging systems is pushbroom that simultaneously with satellite movement, the area covered line to line is captured by the desired resolution. Also, these days using fieldprogrammable gate array (FPGA) [4] as the main core of processor section of the satellite is conventional due 
to having high capabilities, parallel processing, high resistance to spatial radiation, simplicity of reprogramming and more important than all simplicity of software methods appliance to increase reliability and their resistance to single event upsets (SEU) in space. In the following, after analyzing the block diagram more detailed and analyzing the simulated and practical results of each of the sections with creating a test design, image is created at the output.

According to the conditions of imaging, it is possible that the captured image wouldn't have high quality and there would be a need to improve the image quality. One of the important qualitative factors in processing different images is the contrast. Contrast is generated by the difference between the reflected brightness of two adjacent surfaces [5]. General histogram equalization (GHE) is the most common method for contrast enhancement which is used for almost all images due to its simplicity and relatively good performance. The main idea of GHE is to remap the grey levels of the input image based on the uniform distribution of its probability density function (PDF) [6-7]. Also, due to over-enhancement, this method tends to create issues such as intensity saturation, generation of annoying artificial effects and noise amplification in the output image [8-9]. To overcome these limitations, numerous methods are proposed [10-20]. One of the earliest proposed methods was called brightness preserving bi-histogram equalization (BBHE) [10]. In BBHE method, the main histogram is partitioned into two sub-histograms based on the mean intensity values. Then, the equalization is performed separately on each sub-histogram. Then, the recursive meanseparate histogram equalization (RMSHE) method is proposed [13]. In RMSHE method, similar to BBHE, the main histogram is divided into two partitions. These sub-histograms are again divided into two partitions based on their mean values. This process is repeated recursively to reach the desired number of histograms. Another method called recursive sub-image histogram equalization (RSIHE) is presented which is similar to RMSHE [14]. In RSIHE method, the histogram is recursively divided based on the median of intensity values instead of their mean. In the following, the brightness preserving dynamic histogram equalization (BPDHE) method is proposed [21]. In BPDHE method, the local maxima are used as the separating points after applying the Gaussian smoothing filter. Before the equalization process, each sub-histogram is mapped into a new dynamic range. To maintain the mean brightness of the image, normalizing the brightness is performed at the final stage.

Neither of the above-mentioned methods have control on the enhancement ratio. Therefore, a method is presented which is based on histogram clipping called bi-histogram equalization with a plateau limit (BHEPL) [22]. In BHEPL, similar to BBHE, the main histogram is partitioned into two sub-histograms. Then, controlling the enhancement ratio is performed by adjusting the plateau limit as the mean number of the occurrence of intensity for each sub-histogram. Finally, the equalization process is performed independently on each clipped sub-histogram. Then, the dynamic quadrants histogram equalization plateau limit (DQHEPL) method is proposed [23-24]. In DQHEPL method, the main histogram is partitioned into four sub-histograms based on the median of the intensity values. Then, the clipping process is performed in each sub-histogram based on the mean number of the occurrences of intensity. Subsequently, before performing the equalization process, each sub-histogram is mapped into a new dynamic range while the second separating point is maintained as preserving the mean brightness. median-mean based sub-image-clipped histogram equalization (MMSICHE) method is presented as follows [25]. In MMSICHE, the main histogram is initially divided into two partitions based on the median of the intensity values. Then, based on the mean intensity values in each sub-histogram, it is divided into four partitions. Then, the clipping process of the main histogram is carried out based on the median of the number of the occurrences of intensity. Recently, entropy-based dynamic sub-histogram equalizations (EDSHE) is presented [26]. In EDSHE method, the main histogram is initially divided into two sub-histograms with equal entropies. Then, these sub-histograms are again divided into two partitions based on entropy. This process is repeated recursively to reach the optimum number of sub-histograms until satisfying the termination criterion. Before performing the equalization process, each sub-histogram is mapped into a new dynamic range.

Each above-mentioned method is proposed to resolve a specific problem in the field of contrast enhancement. However, methods which could follow multiple targets are barely proposed [27-28]. In this paper, entropy-based triple dynamic clipped histogram equalization (ETDCHE) method is proposed. ETDCHE creates a reasonable and balanced relationship among controlling the enhancement ratio, preserving the mean brightness and conserving the average information content such that a clear image is generated at the output with the maximum details and natural enhancement without unwanted artifacts such as intensity saturation. In this method, the histogram of the input image is initially partitioned based on entropy to three partitions with almost similar number of pixels. Then, in order to control the enhancement ratio, the process of clipping the histogram is then applied with the same threshold level as the mean of occurring intensities in each subhistogram. Then, each sub-histogram is mapped into a new dynamic range and finally, the equalization process is independently performed on each sub-histogram. 


\section{DESIGN, SYSTEMATIC CALCULATIONS AND PRESENTING THE BLOCK DIAGRAM OF THE SATELLITE CAMERA}

In this paper, the aim is to design and fabricate single-spectrum satellite imaging board with 8 meters ground sample distance (GSD) that is supposed to be located at a hypothetical height of 300 kilometers from the ground capturing images from different areas of the earth. Parameters such as orbital height, GSD, Swath and imaging spectral area are specified according to the purpose. Imaging sensor and imaging method parameters can be determined by statistical studies. The number of pixels, focal length, the optical set aperture, electronic shutter rate can be obtained using the calculations that are outlined in the following. The electronic shutter rate related to CCD is considered one millisecond that this time can provide achieving to 8 meters GSD according to the average speed of low altitude satellites $(8 \mathrm{~km} / \mathrm{s})$. This can be feasible with providing an optic with focal length and optical aperture that is obtained from (1), (2) [29]. The proposed characteristics are listed in Table 1. In the following, we describe the general block diagram of the satellite camera and the detailed block diagram of the electronic part with complete details.

$$
\begin{aligned}
& D=2.44 \frac{\lambda H}{G S D}=6.4 \mathrm{~cm} \\
& \mathrm{f}=\frac{\mathrm{Hx}}{\mathrm{GSD}}=52.5 \mathrm{~cm}
\end{aligned}
$$

\subsection{The general block diagram of the satellite camera}

In Figure 1 the general proposed block diagram including 4 main units of optic, detector, processing, and memory is shown. Which communicate with other sections such as power unit and on-board computer $(\mathrm{OBC})$. The collected light from the ground enters the CCD linear sensor by an optic unit that creates an electrical charge in its pixels relating to the amount of energy in incident light. For driving CCD a series of trigger pulses are required which these pulses are different according to different CCDs and for creating them FPGA is used. These pulses enter CCD after crossing a level shifter for providing the required current of CCD from FPGA voltage and voltage level matching of FPGA with the required voltage. The created output from CCD that is an analog voltage enters analog front end (AFE). In an AFE first sampling of the received analog data from CCD is done according to the different modes and after a series of amplifying operations, analog input data is converted to digital output [30]. This component also needs pulses for driving that are obtained by FPGA. some of them are for synchronization clock and some of them are for giving required information for programming internal registers of the analog front end.

These days, the worldwide use of FPGAs as the main core of management and processing the data is conventional. The processor section other than providing The trigger clock of CCD board and clock for synchronization of sampler circuits and programming the internal registers of AFE has the duty of collecting digitalized data. The processor section has the duty of receiving data such as camera turning on and shutting off, image store command and sending the stored image command using telecommand unit, sending data such as detector section temperature and processor section temperature using telemetry unit and sending the image data to telecommunication center. These sections are simulated using a computer and RS232 protocol. Also, this section receives the required power supply from the power supply sector. In memory unit, PROM is used for permanent storage of program FPGA, and FLASH are used for permanent storage of taken images and SRAM is used for temporarily storing the data relating to images in different sections.

Table 1. The proposed characteristics for camera

\begin{tabular}{cc}
\hline parameter & value \\
\hline Height $(\mathrm{H})$ & $300 \mathrm{Km}$ \\
Spectral Band $(\lambda)$ & $0.7 \mu \mathrm{m}$ \\
Ground Sample Distance & $8 \mathrm{~m}$ \\
(GSD) & $\mathrm{CCD}$ \\
Imaging Sensor & Pushbroom \\
Imaging Method & $\mathrm{N}=$ Swath $/ \mathrm{GSD} 2048$ \\
The Number of Pixels (N) & $12.8 \mathrm{Cm}$ \\
The Value of Optical Aperture & $14 \mu \mathrm{m}^{*} 14 \mu \mathrm{m}$ \\
(D) & $52.5 \mathrm{Cm}$ \\
Pixel Pitch (X) & $\mathrm{t}=\mathrm{GSD} / \mathrm{V}=1 \mathrm{~ms}$ \\
Focal Length (f) & $16 \mathrm{~km}^{2}$ \\
Shutter Rate (t) &
\end{tabular}

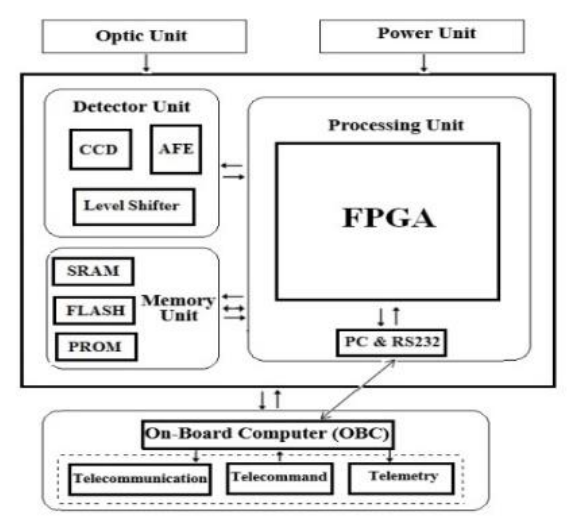

Figure 1. The proposed block diagram of the satellite camera 


\subsection{Detailed block diagram of an electronic section of the satellite camera}

In Figure 2 the detailed block diagram of the electronic section of satellite camera is shown.

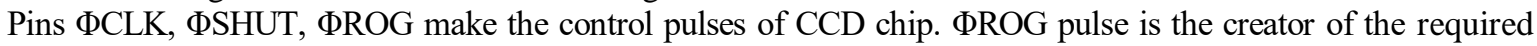
pulse for output reading gate and transfers the accumulated charges in the detector element in parallel by vertical transition gate to horizontal transition gates for sending outside of CCD sensor that this is possible according to the $\Phi C L K$ pulse which is the generator of sensor main clock. With electronically controlling $\Phi S H U T$ pulse lighting operation can be controlled. These pulses have a range of zero to 5 volts. Since FPGA has the capability to create 3.3-volt pulses thus it is required that pulses enter CCD after a level shifter. Finally, the generated output at Vout is transferred to AFE.

Before using AFE the internal registers should be configured. For giving the needed information for programming the internal registers of CONFIG, MUX, PGA and OFFSET pins SDATA, SCLK and SLOAD are used. SCLK pulse as a serial clock pulse for programming the registers, SDATA pulse for reading and writing the needed information for configuring the registers and SLOAD as verifying the information in SDATA pulse. After configuring AFE for driving it needs CDSCLK1, CDSCLK2 and ADCCLK pulses. CDSCLK1 pulse samples the reference level of CCD waveform and CDSCLK2 pulse samples the CCD waveform data level. The difference between these two voltages is shifted according to the offset register and then it is scaled with the adjusted value in PGA. The output voltage of PGA enters ADC and it is sampled there according to ADCCLK clock and sends data to the output as 8 bit.

All of the programming operation in FLASH memory is done based on the page to page process. Also, it has 2 buffers in the size of a page. FLASH programming is done through 3 steps of reading, erasing and writing. Erasing operation can be done as blocks, sectors, whole chip or page surface. Writing operation in memory can be possible by writing in the buffer, writing from buffer to main memory or writing directly on the main memory. Reading from memory can be done through buffers, reading the main memory page and reading the main memory page continuously. For each of these instructions, there is a specific app code and it uses a serial mediator for achieving information. FLASH memory is activated using $\overline{\mathrm{CS}}$ pin and is accessible through a 3 wire mediator that includes serial input (SI), serial output (SO) and serial clock (SCK).

SRAM memory has 19 pins of A0 to A18 for addressing, 8 pins of R-I/O0 to R-I/O7 for writing and reading 8-bit data and 3 control pins of R-WE R-OE and R-CS that with their adjustment various modes of writing and reading is provided. The simulation of connection of processor part of the camera with telemetry, telecommand, and telecommunication is done using MAX232 and DB9 port. This component provides the possibility of synchronous bilateral communication. The input of MAX receiver from receive pin is connected to PC and RXD output is connected directly to FPGA while the input of TXD transceiver from FPGA and the output enters PC from transmitting pin. In serial communication, the data is sent bit by bit. Therefore, before sending it should become in serial form and be sent in an 8-bit packet.

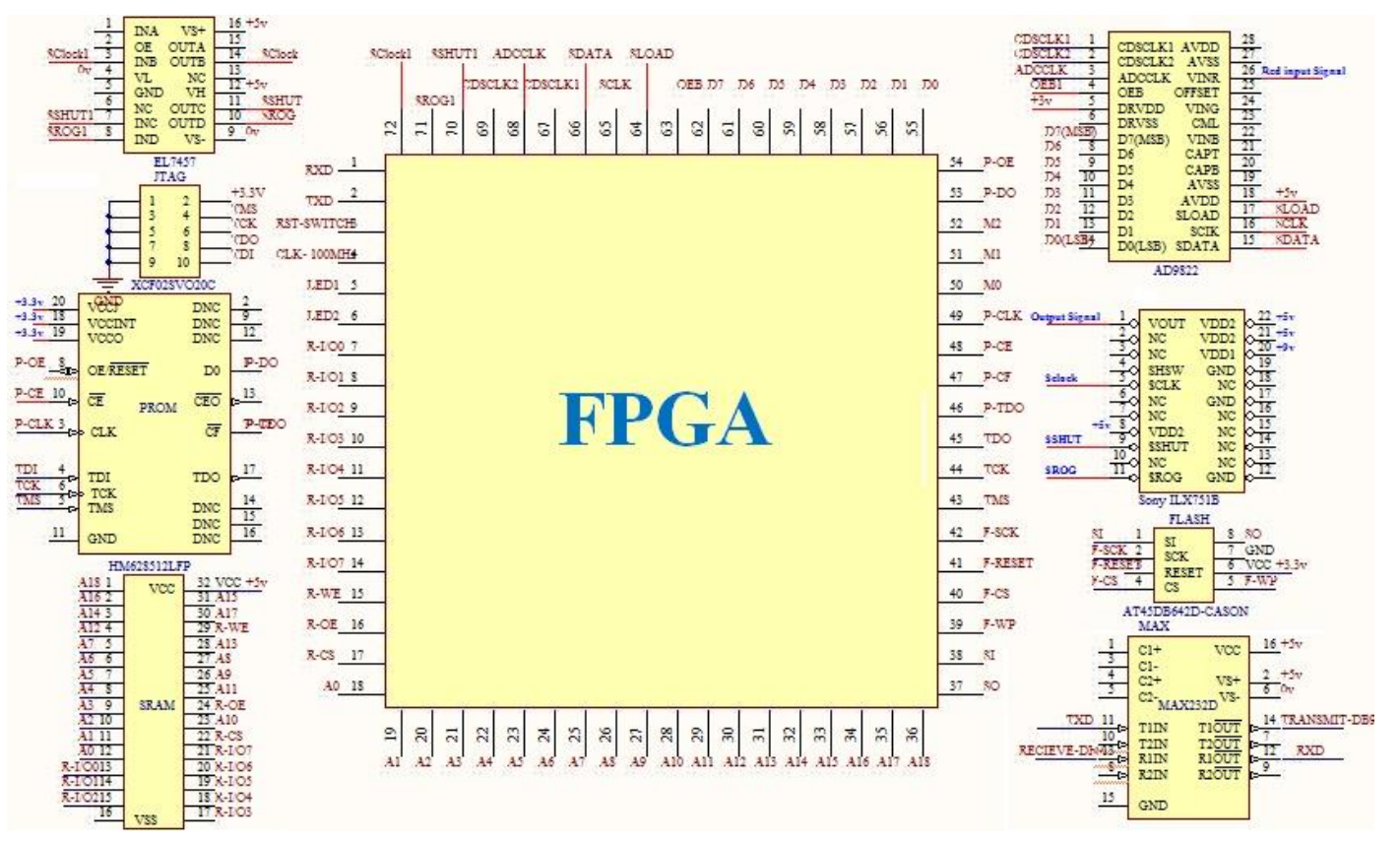

Figure 2. Detailed block diagram of the electronic part of the satellite camera 
FPGA as the main core of the processor has duties such as receiving the digitalized output of AFE, generating the trigger pulses of CCD and AFE, temporarily storing data in SRAM, storing data permanently in FLASH memory, communicating with PROM for permanently saving FPGA program and communicating with RS232 connector for simulating subsystems of telecommand, telemetry and telecommunication unit. The connection of PROM memory to FPGA is established for permanently storing the program through separate configuration pins and JTAG pins.

\section{IMPLEMENTATION OF THE ELECTRONIC PART}

In this section, description, and explanation of electronic part including work modes of the camera, simulation results and practical results are shown.

\subsection{Working modes of the camera}

In the implementation of the electronic part of camera 3 operational modes are considered below that according to the received command from telecommand camera works in one of these 3 modes. Real-time mode: this mode starts working when the camera is in the vision of ground station and condition for imaging is suitable and sends the captured images directly through the telecommunication unit. In this mode, we can capture images in the whole time of presence of satellite in the vision of ground station and there is no limit for the size of image transmission. In this mode, images are stored temporarily in SRAM and then are read out for sending. Storage mode: this mode starts working when the camera is not in the vision of ground station and the condition for imaging is suitable and the captured images are saved in FLASH permanently after temporarily being saved on SRAM. In this mode, according to the capacity of memory, the images can be saved. Storage-send mode: in this mode when the camera is in the vision of ground station and the required command is issued from ground station stored images in FLASH are read out and are sent to ground through telecommunication unit.

\subsection{Simulation results and practical implementation}

In this section, the results of practical implementation and the results of simulation of programs written by VHDL coding language in Xilinx ISE software (version 14.1) and the serial port of code -vision software are brought. In Figures 3(a) and 4(a) pulses (ROG and ФCLK are shown for driving CCD. The period of $\Phi C L K$ pulse is 480ns according to the shutter rate of $1 \mathrm{~ms}$ and 2087 pixels of CCD that is created by the clock pulse of the external crystal. In Figure 3(b) configuring the offset register with the number 0101111001100101 as one of four internal registers of AFE is shown. Each AFE channel has an exclusive offset adjustment. In the pulse, first zero bit of SDATA represents writing in registers and the address 101 for adjusting red OFFSET and 3 ineffective 1 bit and in the following bits D8 to D0 controls the offset range from $-350 \mathrm{mv}$ to $+350 \mathrm{mv}$ in 512 different positions. In all this time SLOAD pulse is in zero state. After configuring AFE in Figures 3(c) and 4(b) CDSCLK1, CDSCLK2 and ADCCLK pulses in order to drive AFE for creating data are shown as 8 bits. Thus it is required that pulses with 3.3-volt range (created by FPGA) is converted to a pulse with 5-volt range (after crossing the level shifter) for entering CCD.

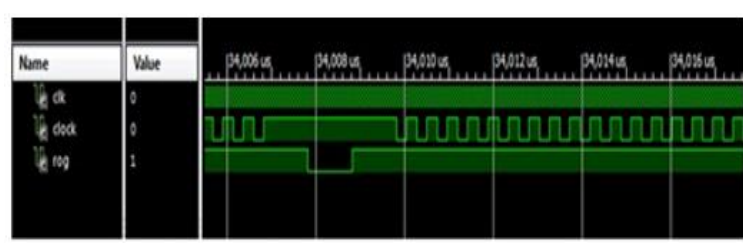

(a)

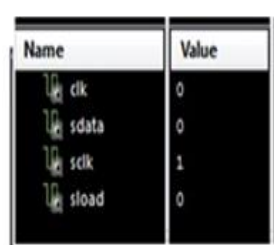

b sload

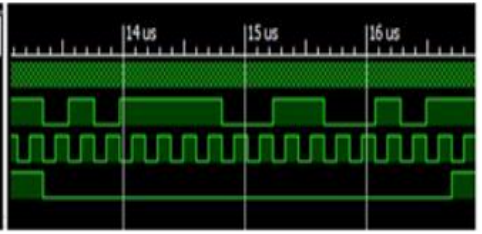

(b)

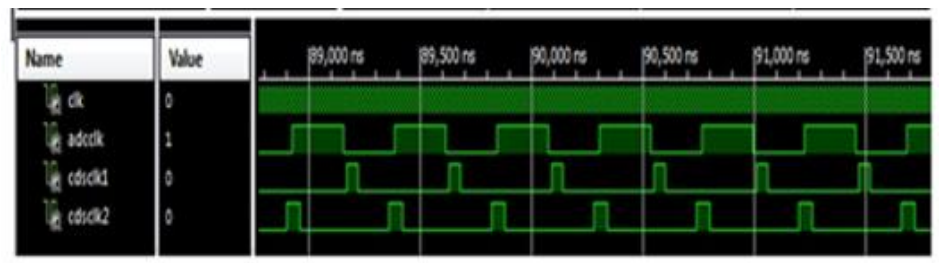

(c)

Figure 3. Simulation results (a) CCD driving (b) AFE configuration (c) AFE driving in ISE 


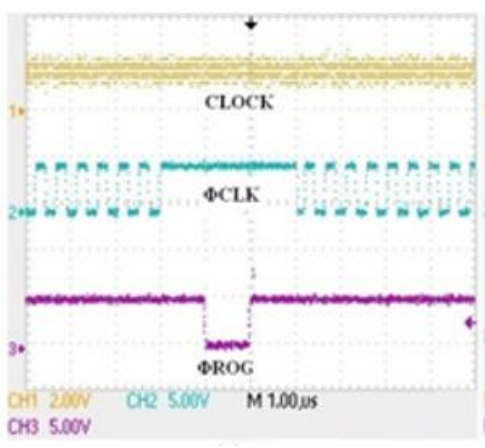

(a)

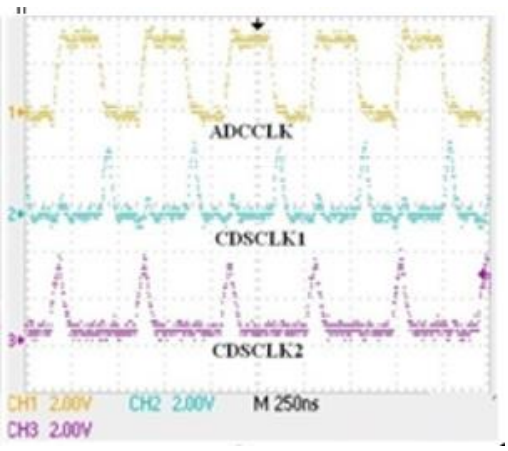

(b)

Figure 4. Practical results (a) CCD driving (b) AFE driving

As it is mentioned before one of the types of writing inside memory is writing directly in the main memory that is a composition of two operations of writing in buffer and writing from buffer to the main memory. The operation starts with the falling edge of $\overline{\mathrm{CS}}$ and with clocking of SCK through serial input (SI), instruction specific app code in this state according to the choice of buffer 1 or 282 or 85 HEX code is sent to the memory. Then, 3 address bytes including 13 bit of desired page address and 11 bits of the number of the byte which writing should be started from there are sent. After sending these bits, data is read from the input and is written in buffer. With $\overline{\mathrm{CS}}$ becoming one, first the desired page is cleared and data is written on it from the buffer. For directly reading from a page of the main memory first a D2 HEX code should be sent to the memory and 3 address bytes are sent including 13 bits of the desired address page and 11 bits of the number of bytes which reading should be started from there. After sending these bits, 4 ineffective bits should be sent that their being zero or one is not important. $(\overline{\mathrm{CS}})$ pin should be zero in all these steps. After sending all of the bits, the desired data is placed at the output. The bytes of the desired page are sent until the page finishes. For instance, 4 data bytes of 01110111101010100100010010001000 in the 528th byte of page 528 with SCK clock with $50 \mathrm{MHz}$ frequency made from the clock of $100 \mathrm{MHz}$ crystal are written and again is read out from the same page and is shown through the serial terminal of the computer. Figure 5(a) shows the direct writing simulation Figure 5(b) shows the direct reading simulation and Figure 5(c) shows the output of the serial terminal in the computer.

Writing operation in SRAM includes steps of OE pin becoming one, uploading address in address pins, setting CS and WE to zero, uploading data on I/O pins and finally setting CS and WE to one. Also, reading operation in SRAM includes uploading address on its pins, setting CS and OE to zero, reading data from I/O pins and finally setting CS and OE to one. For example, 1-byte data of 00101000 is written in the binary address of 0000000000010000000 and equivalent of 128 decimal with CLK clock equal to $100 \mathrm{MHz}$ crystal clock and again it is read from the same address. Figure 6(a) shows the writing simulation in SRAM and Figure 6(b) shows the reading simulation from SRAM.

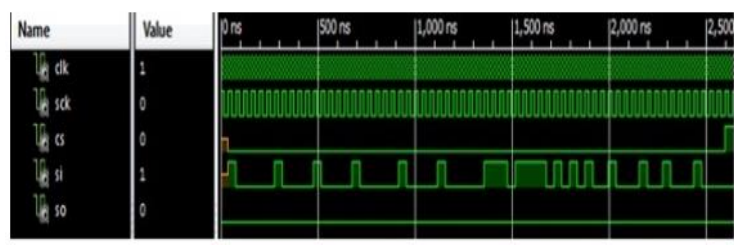

(a)
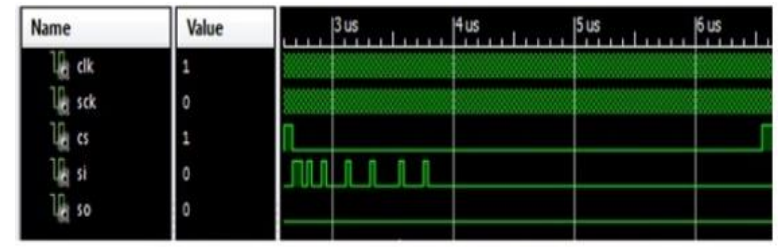

(b)

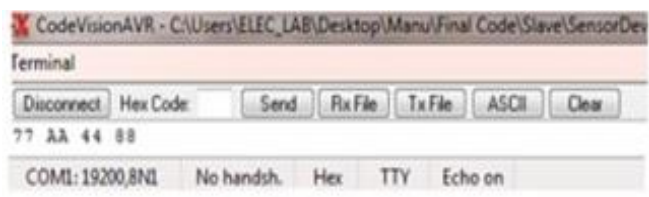

(c)

Figure 5. Simulation results (a) directly writing in flash (b) directly reading from the flash (c) the serial terminal output in ISE software 


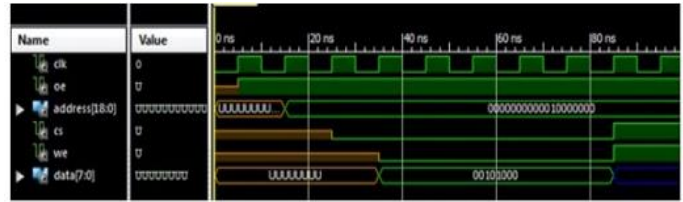

(a)

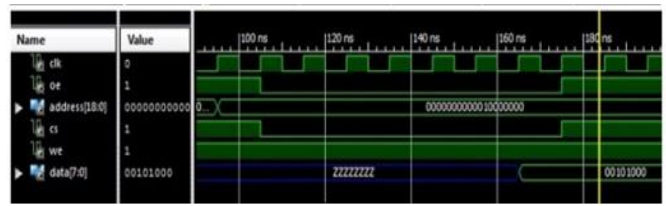

(b)

Figure 6. Simulation results (a) writing in SRAM (b) reading from SRAM in ISE software

\section{ENTROPY-BASED TRIPLE DYNAMIC CLIPPED HISTOGRAM EQUALIZATION}

The proposed ETDCHE method is consisted of four main process including partitioning, clipping, mapping and equalization. In the following, the proposed method is subsequently fully explained in details.

\subsection{Triple partitioning of the histogram}

In the previous method, the histogram of the input image is partitioned into multiple sub-histograms using the mean, median, maxima, minima, exposure level, and entropy values. In methods that use the maxima and minima points for histogram partition, significant valley or peaks should exist in the histogram of the input image. On the other hand, in case of using the mean and median for partitioning the histogram into two partitions, abnormal enhancement might occur for some regions such as in general histogram equalization method. Also, using the mean, median and entropy, recursively, which divides the histogram into four or more partitions might not lead to considerable enhancement on some parts. Also, finding the optimum recursive level is a major challenge in these methods. Therefore, to overcome these challenges, the proposed method divides the histogram of the input image into three partitions by inspiring from entropy-based partitioning such that the number of pixels in each sub-histogram is almost equal. This type of portioning leads to natural enhancement, maximum average information content and preserving reasonable brightness in the output image. Triple partitioning of the histogram is consisted of the following two steps. At the first step, PDF [7] and entropy [31-34] of the input image are achieved using (3) and (4).

$$
\begin{aligned}
& \operatorname{PDF}(\mathrm{i})=\frac{\mathrm{h}(\mathrm{i})}{\mathrm{N}} \quad 0 \leq \mathrm{i} \leq \mathrm{L}-1 \\
& \mathrm{E}(\mathrm{i})=-\sum_{\mathrm{i}=0}^{\mathrm{L}-1} \mathrm{PDF}(\mathrm{i}) * \log _{2} \operatorname{PDF}(\mathrm{i})
\end{aligned}
$$

In the equations above, $\mathrm{h}(\mathrm{i})$ is the histogram function, $\mathrm{N}$ is the total number of pixels in the input image and L-1 is the highest intensity value of the pixels in $n$-bit image $\left(2^{n}=\mathrm{L}-1\right)$.In the second step, the separating points of $\mathrm{m}_{1}$ and $\mathrm{m}_{2}$ are set to be at the intensity levels at which the entropy values at those intensity levels are equal to 0.33 and 0.66 of the entropy at intensity level L-1, respectively.

$$
\begin{array}{ll}
\mathrm{m}_{1}=\mathrm{i} \text { where } & \mathrm{E}(\mathrm{i}) \cong \frac{1}{3} \mathrm{E}(\mathrm{L}-1) \\
\mathrm{m}_{2}=\mathrm{i} \text { where } & \mathrm{E}(\mathrm{i}) \cong \frac{2}{3} \mathrm{E}(\mathrm{L}-1)
\end{array}
$$

Therefore, if the histogram of the input image has intensity range $\left[\mathrm{m}_{0}: \mathrm{m}_{3}\right]$ before partitioning process, where $m_{0}$ and $m_{3}$ are the lowest and highest intensity values in the input image, respectively, the histogram of the input image $\mathrm{W}$ in the proposed ETDCHE method is divided into three sub-histograms with almost the same number of pixels called $\mathrm{W}_{\mathrm{L}}, \mathrm{W}_{\mathrm{M}}$, and $\mathrm{W}_{\mathrm{U}}$. The intensity ranges of each sub-histogram are obtained to be $\left[\mathrm{m}_{0}: \mathrm{m}_{1}-1\right],\left[\mathrm{m}_{1}: \mathrm{m}_{2}-1\right]$, and $\left[\mathrm{m}_{2}: \mathrm{m}_{3}\right]$, respectively. Figure $7(\mathrm{a})$ shows an example of triple partitioning.

\subsection{Histogram clipping}

The main idea in histogram clipping is that it controls the enhancement ratio in the equalization process such that the occurrence of intensity saturation and over-enhancement in the enhanced image is prevented which eventually leads to the generation of natural appearance in the enhanced image. Therefore, in the clipped histogram, the shape of the input histogram changes based on a specific threshold level before the equalization process. Bars of the histogram which have areas larger than the threshold level are bounded by the threshold level. Histogram clipping which is carried out in ETDCHE method is such that the mean number of the occurrences of intensity in each sub-histogram is considered as the clipping threshold level of that sub-histogram [22]. The clipping threshold level of each sub-histogram is determined by (7). 


$$
\mathrm{T}_{\mathrm{j}}=\frac{1}{\mathrm{~m}_{\mathrm{j}}-\mathrm{m}_{\mathrm{j}-1}} \sum_{\mathrm{i}=\mathrm{m}_{\mathrm{j}-1}}^{\mathrm{m}_{\mathrm{j}}-1} \mathrm{~h}(\mathrm{i}) \quad \& \quad 1 \leq j \leq 3
$$

In the equation above, $h(i)$ is the histogram of the input image and $T_{1}, T_{2}$, and $T_{3}$ are the clipping thresholds determined for all three sub-histograms. In Figure 7(b), the calculated clipping thresholds for each sub-histogram could be observed. The clipped histogram is determined by (8).

$$
\mathrm{h}_{\mathrm{c}}(\mathrm{i})=\left\{\begin{array}{ll}
\mathrm{h}(\mathrm{i}) & \mathrm{h}(\mathrm{i})<\mathrm{T}_{\mathrm{j}} \\
\mathrm{T}_{\mathrm{j}} & \mathrm{h}(\mathrm{i}) \geq \mathrm{T}_{\mathrm{j}}
\end{array}\right\} \quad \mathrm{m}_{\mathrm{j}-1} \leq \mathrm{i}<\mathrm{m}_{\mathrm{j}} \& \quad 1 \leq j \leq 3
$$

In the equation above, $h_{c}(k)$ denotes the clipped histogram. Therefore, the clipping method used in ETDCHE causes the enhancement ratio to be controlled throughout the histogram. In Figure 7(c), the clipped histogram is illustrated.

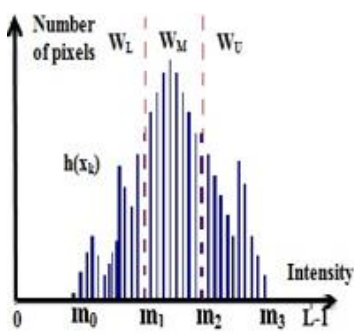

(a)

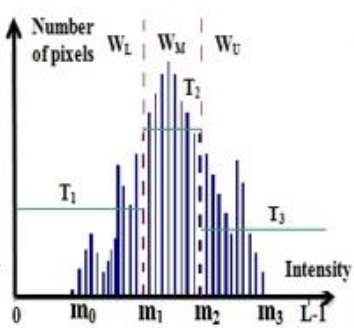

(b)

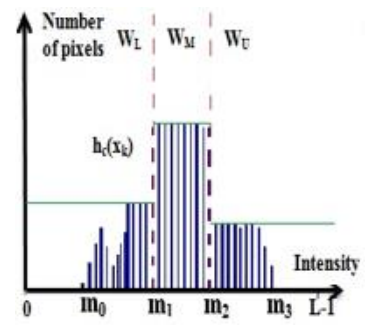

(c)

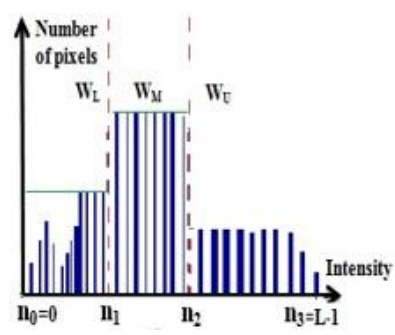

(d)

Figure 7. An example of the proposed ETDCHE method, (a) triple partitioning, (b) calculation of the clipping threshold levels, (c) the clipped histogram, (d) mapping process in to a new dynamic range

\subsection{Mapping each partition into a new dynamic range}

As explained in the partitioning section, each sub-histogram has a specific intensity range. In case of independent equalization of each part, each sub-histogram cannot perform the equalization and histogram expansion process more than the specified range for them. In most cases, sub-histograms with low ranges cannot be enhanced significantly due to lack of sufficient area for enhancement. On the other hand, in subhistograms with high ranges, the issue of over-enhancement comes up.

In order to create a balance in the equalization process and to overcome the above-mentioned issues, ETDCHE method performs the mapping based on the dynamic range of the grey area used in each subhistogram by inspiring from the dynamic histogram equalization method and allocates a new dynamic range to each sub-histogram [17]. However, unlike the dynamic histogram equalization method in which applying the number of pixels in each sub-histogram complicates the computations, in ETDCHE method, no significant impact is observed on the computations of the new dynamic range since the number of pixels are almost the same for each sub-histogram. To simplify the mapping steps, one could ignore the number of pixels in the calculations. The mapping process in ETDCHE method is carried out using (9) and (10).

$$
\begin{aligned}
& \mathrm{n}_{1}=(\mathrm{L}-1) \frac{\mathrm{m}_{1}-\mathrm{m}_{0}}{\mathrm{~m}_{3}-\mathrm{m}_{0}} \\
& \mathrm{n}_{2}=(\mathrm{L}-1) \frac{\mathrm{m}_{2}-\mathrm{m}_{1}}{\mathrm{~m}_{3}-\mathrm{m}_{0}}+\mathrm{n}_{1}
\end{aligned}
$$

The lowest and highest intensity values of the pixels in the output image are considered to be $\mathrm{n}_{0}=0$ and $\mathrm{n}_{3}=\mathrm{L}-1$, respectively. Therefore, after the mapping process, the new dynamic ranges for the first, second and third sub-histograms change to $\left[n_{0}: n_{1}-1\right],\left[n_{1}: n_{2}-1\right]$, and $\left[n_{2}: n_{3}\right]$, respectively. The mapping process to the new dynamic range is illustrated in Figure $7(\mathrm{~d})$.

\subsection{Equalizing each partition independently}

After determining the new dynamic range for each sub-histogram at the final stage of ETDCHE method, equalization of each partition should be carried out independently. The equalization process of each sub-histogram consists of four steps. In the first step, the PDF associated with each sub-histogram should be 
determined using (11) [7]. In this equation, $\mathrm{N}_{\mathrm{j}}$ is the total number of pixels in each clipped sub-histogram which is determined by (12).

$$
\begin{aligned}
& \operatorname{PDF}_{\mathrm{j}}(\mathrm{i})=\frac{\mathrm{h}_{\mathrm{c}}(\mathrm{i})}{\mathrm{N}_{\mathrm{j}}} \quad \mathrm{m}_{\mathrm{j}-1} \leq \mathrm{i}<\mathrm{m}_{\mathrm{j}} \quad \& \quad 1 \leq j \leq 3 \\
& \mathrm{~N}_{\mathrm{j}}=\sum_{\mathrm{i}=\mathrm{m}_{\mathrm{j}-1}}^{\mathrm{m}_{\mathrm{j}}-1} \mathrm{~h}_{\mathrm{c}}(\mathrm{i}) \quad \& 1 \leq j \leq 3
\end{aligned}
$$

In the second step, the cumulative density function (CDF) of each sub-histogram is defined using the corresponding PDF [7].

$$
\operatorname{CDF}_{\mathrm{j}}(\mathrm{i})=\sum_{\mathrm{i}=\mathrm{m}_{\mathrm{j}-1}}^{\mathrm{m}_{\mathrm{j}}-1} \operatorname{PDF}_{\mathrm{j}}(\mathrm{i}) \quad \& 1 \leq j \leq 3
$$

In the third step, the transfer function of each sub-histogram is determined using the CDF associated with each sub-histogram according to (14). Using the obtained transfer functions, each partition is equalized independently. The fourth step includes integrating all three sub-histograms to create a complete image. Therefore, the output image of ETDCHE method is generated by combining all of the three transfer functions.

$$
\mathrm{F}_{\mathrm{j}}=\mathrm{n}_{\mathrm{j}-1}+\left(\mathrm{n}_{\mathrm{j}}-1-\mathrm{n}_{\mathrm{j}-1}\right) * \mathrm{CDF}_{\mathrm{j}} \quad \& 1 \leq j \leq 3
$$

\section{SIMULATION RESULTS AND DISCUSSION}

In this section, first, the method of testing the camera is described. Then the evaluation of the quality of the received images in different lighting conditions before and after enhancement with ETDCHE method is discussed. In the following, the simulation results of the proposed ETDCHE method are compared with the proposed histogram equalization-based methods e.g. GHE [7], BBHE [10], RMSHE [13], RSIHE [14], BPDHE [21], BHEPL [22], DQHEPL [24] and MMSICHE [25]. For better assessment and comparison of the methods, nature and road images with histograms focused on the dark side, tank and aircraft images with histograms focused on the middle side and snowy mountains and beach images with histograms focused on the bright side are used as test images.

\subsection{The method of testing the camera and evaluation of the received images quality}

Due to the fact that in this paper the aim is fabricating and testing the electronic part of an imaging system, an optic one lens system with characteristics of focal length $18 \mathrm{~cm}$ and aperture diameter of $5 \mathrm{~cm}$ for receiving image was provided. In the following, we place the image A at $180 \mathrm{~cm}$ distance of the lens. With these conditions according to (2) resolution for two distances of $300 \mathrm{~km}$ and $180 \mathrm{~cm}$ is $23.3 \mathrm{~m}$ and $140 \mu \mathrm{m}$, respectively. For simulating the linear sweep system, image A should pass in front of the camera with the speed of $\mathrm{V}=\mathrm{GSD} / \mathrm{t}=0.14 \mathrm{~m} / \mathrm{s}$. For verifying the captured pictures, we save 700 pixels as image $\mathrm{B}$. If we multiply 700 pixels by $140 \mu \mathrm{m}$ resolution $9.8 \mathrm{~cm}$ is obtained. This number is equal to the part of image A which is saved in image B. This verification is true in all of the captured images. In Figure 8 the general view of the camera is shown. Of course, with providing an optic with the proposed characteristics of Table 1 we can achieve to 8-meter resolution in $300 \mathrm{~km}$ distance.

After providing the aforementioned conditions in the above section, the images of Figure 9 are chosen as main images for testing the camera and are moving in front of the camera through the linear sweep system. Figures 10 and 11 illustrate the received images of camera in different imaging conditions from left to right, normal, high, and low lighting conditions. In the upper part of these figures, the received images from the camera before enhancing the contrast and in the lower part the received images after the enhancement are shown. The obtained results from the visual comparison in some of the viewers show that the quality of the received images after the enhancement is improved significantly and indicates that contrast enhancement has largely overcome the unfavorable lighting conditions during imaging. 


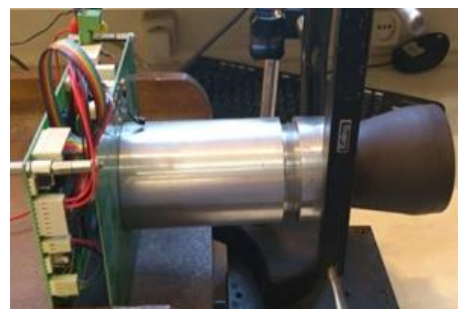

Figure 8. The general view of the camera
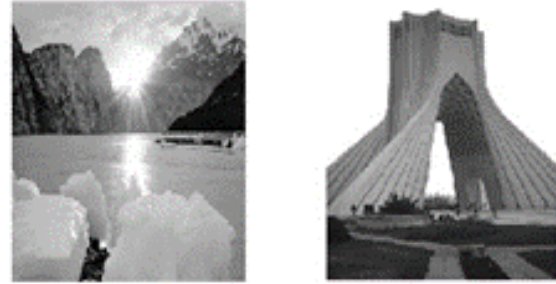

Figure 9. the main images for testing the camera

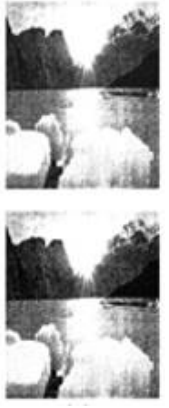

(a)

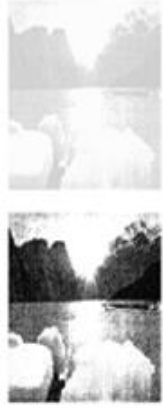

(b)

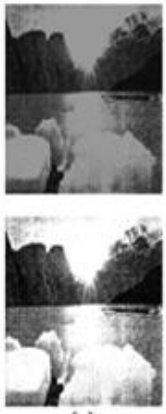

(c)

Figure 10. Received images in lighting conditions a) normal b) high c) low, the upper part is before enhancement and the lower part is after enhancement
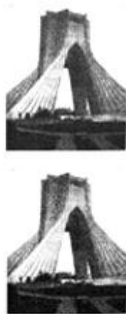

(a)
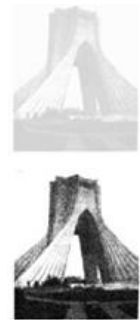

(b)
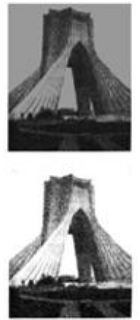

(c)

Figure 11. Received images in lighting conditions a) normal b) high c) low, the upper part is before enhancement and the lower part is after enhancement

\subsection{Performance assessment based on average information content}

Among the contrast enhancement method, the most appropriate method is the one which could show all the information of the input image besides natural contrast enhancement. Therefore, to show the capability of the proposed ETDCHE method in extracting the information from an image, one could use the average information content (entropy) as a quantitative assessment. Entropy measures the richness of the details of the image and it is usually measured in bits. Higher entropy values show more available information in the image. The definition of entropy is expressed in (4). However, for a better visual perception from the image, one needs redundant information besides actual information (entropy). The proposed algorithm leads to enhancements in visual quality by changes in the type and volume of redundant information. To achieve optimum performance, the entropy should be close to the original image to maintain the actual information of the image as well. In Table 2, the entropy results are listed using different method for each six images. Based on the Table 2, the proposed method generates the highest entropy for all the images compared to other methods such that the generated entropy for all of the images is very close to that of the original image. Therefore, due to high entropy which is close to the original image, the proposed method is the best method to extract the information content in the image. Also, it guarantees natural enhancement in the output image.

Implementation of the electronic sector of the satellite camera and image contrast ... (Majid Zarie) 


\begin{tabular}{|c|c|c|c|c|c|c|c|c|c|c|}
\hline Method & INPUT & GHE & BBHE & RMSHE & RSIHE & BPDHE & BHEPL & DQHEPL & MMSICHE & ETDCHE \\
\hline Figure 12 & 4.655 & 4.518 & 4.459 & 4.504 & 4.522 & 3.997 & 4.508 & 4.58 & 4.652 & 4.653 \\
\hline Figure 13 & 4.607 & 4.469 & 4.504 & 4.303 & 4.303 & 4.059 & 4.531 & 4.571 & 4.606 & 4.606 \\
\hline Figure 14 & 5.645 & 4.957 & 5.537 & 5.508 & 5.483 & 5.396 & 5.613 & 5.58 & 5.599 & 5.627 \\
\hline Figure 15 & 4.005 & 3.747 & 3.924 & 3.965 & 3.947 & 3.938 & 3.994 & 3.994 & 3.992 & 3.996 \\
\hline Figure 16 & 5.939 & 5.245 & 5.804 & 5.814 & 5.807 & 3.998 & 5.856 & 5.847 & 5.908 & 5.918 \\
\hline Figure 17 & 5.396 & 5.385 & 5.379 & 5.307 & 5.3 & 3.524 & 5.385 & 5.385 & 5.385 & 5.396 \\
\hline
\end{tabular}

\subsection{Performance assessment based on visual quality}

In addition to qualitative assessment using the average information content, qualitative assessment of contrast enhancement is necessary as well. The objective of qualitative assessment is to discuss and evaluate the effects of annoying artifacts, over-enhancement and natural appearance in the enhanced images. Therefore, using the visual assessment results, the performance of different methods are compared based on qualitative criteria. Analyzing the visual observations using Figures 12 to 17 shows that GHE method highly tends to create intensity saturation and noise amplification in the output image, due to sudden change in the intensity levels; also, due to lack of proper control on the enhancement, unnecessary visual destructive effects are observed in the images with this method. In BBHE, RSIHE and RMSHE methods, image contrast is partially enhanced. But, the lack of significant enhancement in some regions leads to the loss of details in the images. One of the major reasons for this fact is that the mapping is not carried out for the sub-histograms which cause each sub-histogram not to perform the equalization and histogram expansion processes exceeding the specified range for it.

In BPDHE method, brightness normalizing ratio has a major impact on the output image. In Figure 12 and Figure 13, low ratio leads to negligible contrast enhancement and in Figures 16 and 17, enhancement ratio higher than one leads to exceeding of some the intensities of the pixels than the maximum intensity of the dynamic range of the output and intensity saturation is observed for some parts. In BEHPL, DQHEPL and MMSICHE methods, intensity saturation and unnatural amplification are observed despite high mean entropy values. This is due to the fact that in some parts, significant enhancements are not made leading to the loss of details of intensity saturation. The reasons could be not performing the mapping process in sub-histograms in BHEPL and MMSICHE methods and preserving the second separating point in DQHEPL method.

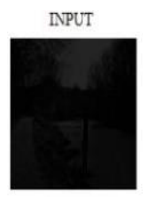

BPDHE
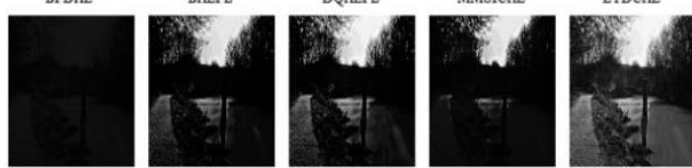

Figure 12. Nature image with histogram focused on the dark side
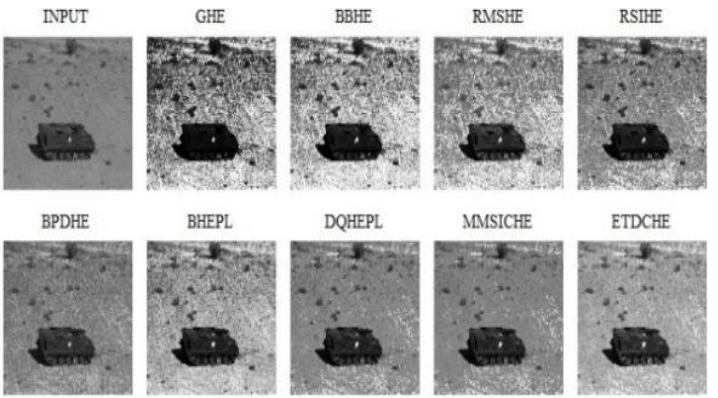

DQHEPL.

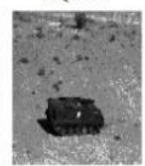

MMSICHE
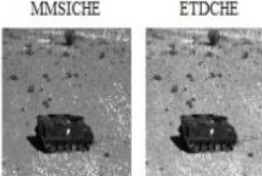

Figure 14. Tank image with histogram focused on the middle range
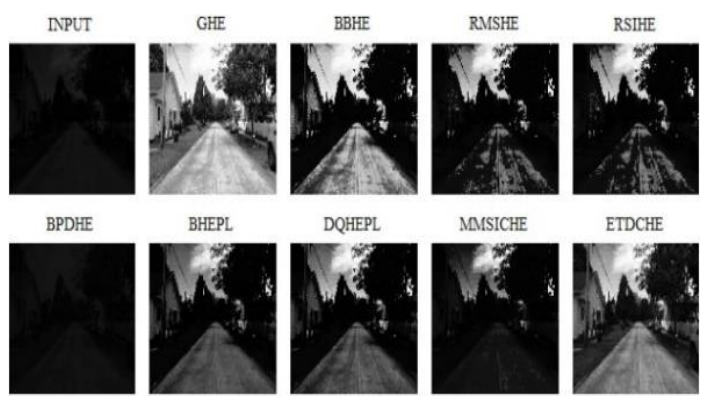

DQHEPL

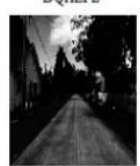

MMSICHE

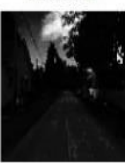

ETDCHE

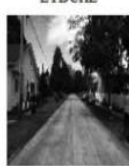

Figure 13. Road image with histogram focused on the dark side

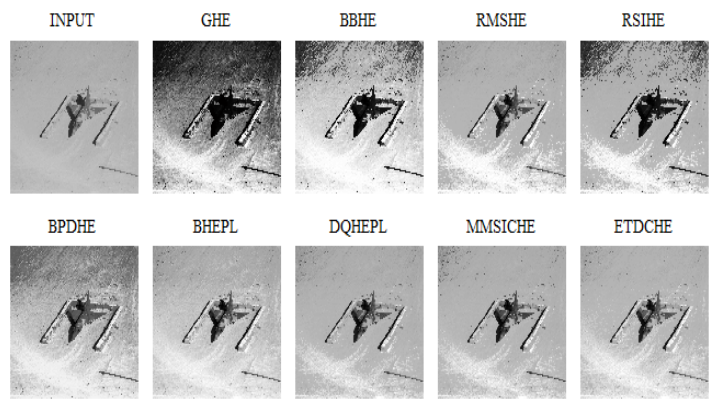

Figure 15. Aircraft image with histogram focused on the middle range 

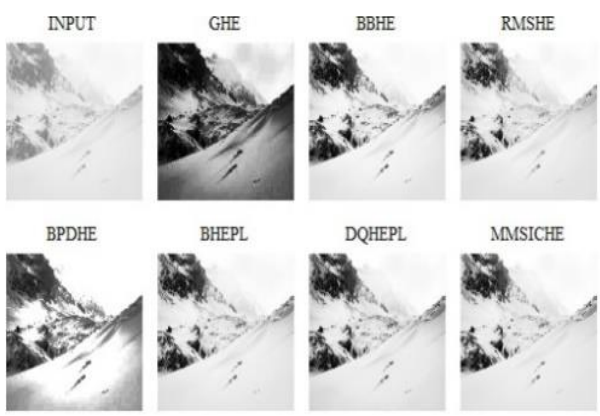

Figure 16. Snowy mountain image with histogram focused on the bright range

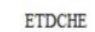

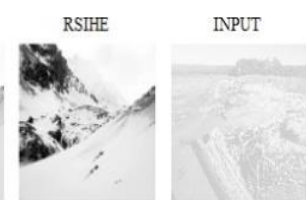
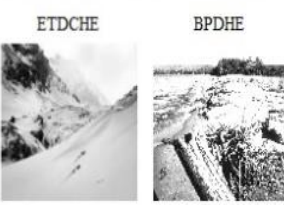

Figure 17. Beach image with histogram focused on the bright range

By observing Figures 12 to 17 one could find out that the proposed ETDCHE method has the best performance compared to other methods. In all of the images, the contrast is successfully enhanced while a reasonable brightness is maintained and natural enhancement is achieved. Therefore, in all of the images, besides expanding the histogram throughout the intensity levels, controlling the enhancement ratio and keeping the details at all levels are observed.

\section{CONCLUSION}

In this paper, the design and implementation of a satellite camera imaging board on the basis of systematic calculations and presenting the general and detailed block diagram of the imaging board including 4 main units of optic, detector, processing, and memory is done. Also with the considered elements for memory unit, this camera can work in three modes of real-time, store and store-sending. The verification of the practical and simulation results is shown according to the received images in this way that image $\mathrm{A}$ is passed in front of the camera with $0.14 \mathrm{~m} / \mathrm{s}$ speed with linear sweep and $\mathrm{n}$ pixels are stored in image B. IF $\mathrm{n}$ pixel is multiplied by $140 \mu \mathrm{m}$ resolution we achieve to number $\mathrm{L}$ which is the part of image A that is saved in image B. According to the conditions of the satellite imaging, in most cases, there is a need to improve the image quality. A very important feature in the satellite images is contrast enhancement. In the following, a powerful method called ETDCHE is proposed to reach multiple targets. The results of the simulations on different images show that the proposed method is the best method for extracting the information content considering its high entropy. Furthermore, in all of the images, besides expanding the histogram throughout the intensity levels, contrast enhancement is successfully achieved such that clear images are created in the output with the maximum details and natural enhancement.

\section{REFERENCES}

[1] J. Fischer and T. Radil, "DSP based measuring line-scan ccd camera," Second IEEE International Workshop on Intelligent Data Acquisition and Advanced Computing Systems: Technology and Applications, 2003. Proceedings, Lviv, pp. 345-348, 2003.

[2] B. Li, J. Jin, L. He, J. Liu and Y. Shang, "Electronics design for an interline CCD camera based on FPGA," Electrical Power Systems and Computers, vol. 99, pp. 463-469, 2011

[3] C. Chengtao, W. Mingyan and L. Yanhua, "FPGA-based array CCD sensor drive system design and implementation," Sensors \& Transducers, vol. 176, no. 8, pp. 49-57, 2014.

[4] T. M. Bittibssi1, G. I. Salama, Y. Z. Mehaseb and A. E. Henawy, "Image enhancement algorithms using FPGA," 2012 8th International Computer Engineering Conference (ICENCO), Cairo, pp. 96-99, 2012.

[5] H. Demirel, C. Ozcinar and G. Anbarjafari, "Satellite image contrast enhancement using discrete wavelet transform and singular value decomposition," in IEEE Geoscience and Remote Sensing Letters, vol. 7, no. 2, pp. 333-337, 2010.

[6] H. Demirel, G. Anbarjafari, and M. N. S. Jahromi, "Image equalization based on singular value decomposition," 200823 rd International Symposium on Computer and Information Sciences, Istanbul, pp. 1-5, 2008.

[7] R. C. Gonzalez and R. E. Woods, "Digital Image Processing, Englewood Cliffs," NJ: Prentice Hall, 2008.

[8] T. Kim, and J. Paik, "Adaptive contrast enhancement using gain controllable clipped histogram equalization," in IEEE Transactions on Consumer Electronics, vol. 54, no. 4, pp. 1803-1810, 2008

[9] L. Zeng, B. Yan, W. Wang, "Contrast Enhancement Method Based on Gray and Its Distance Double-Weighting Histogram Equalization for 3D CT Images of PCBs," Computational Intelligence in Image Processing, vol. 2016, pp. 1-13, 2016.

[10] Y. T. Kim, "Contrast enhancement using brightness preserving bi-histogram equalization," in IEEE Transactions on Consumer Electronics, vol. 43, no. 1, pp. 1-8, 1997. 
[11] Y. Wan, Q. Chen and B. M. Zhang, "Image enhancement based on equal area dualistic sub-image histogram equalization method," in IEEE Transactions on Consumer Electronics, vol. 45, no. 1, pp. 68-75, 1999.

[12] S. D. Chen and A. R. Ramli, "Minimum mean brightness error bi-histogram equalization in contrast enhancement," in IEEE Transactions on Consumer Electronics, vol. 49, no. 4, pp. 1310-1319, 2003.

[13] S. D. Chen and A. R. Ramli, "Contrast enhancement using recursive mean separate histogram equalization for scalable brightness preservation," in IEEE Transactions on Consumer Electronics, vol. 49, no. 4, pp. 1301-1309, 2003.

[14] K. S. Sim, C. P. Tso, and Y. Y. Tan, "Recursive sub-image histogram equalization applied to gray-scale images," Pattern Recognition Letters, vol. 28, no. 10, pp. 1209-1221, 2007.

[15] M. Kim and M.G. Chung, "Recursively separated and weighted histogram equalization for brightness preservation and contrast enhancement," in IEEE Transactions on Consumer Electronics, vol. 54, no. 3, pp. 1389-1397, 2008.

[16] K. Wongsritong, K. Kittayaruasiriwat, F. Cheevasuvit, K. Dejhan, and A. Somboonkaew, "Contrast enhancement using multipeak histogram equalization with brightness preserving," IEEE. APCCAS 1998. 1998 IEEE Asia-Pacific Conference on Circuits and Systems. Microelectronics and Integrating Systems. Proceedings (Cat. No.98EX242), Chiangmai, Thailand, pp. 455-458, 1998.

[17] M. A. A. Wadud, M. H. Kabir, M. A. A. Dewan, and O. Chae, "A dynamic histogram equalization for image contrast enhancement," in IEEE Transactions on Consumer Electronics, vol. 53, no. 2, pp. 593-600, 2007.

[18] T. Celik, and T. Tjahjadi, "Automatic image equalization and contrast enhancement using Gaussian mixture modelling," in IEEE Transactions on Image Processing, vol. 21, no. 1, pp. 145-156, 2012.

[19] C. C. Ting, B. F. Wu, M. L. Chung, C. C. Chiu, Y. C. Wu, "Visual Contrast Enhancement Algorithm Based on Histogram Equalization,” Sensors, vol.15, no.7, pp.16981-16999, 2015.

[20] S. Pearl Mary and V. Thanikaiselvan, "Unified adaptive framework for contrast enhancement of blood vessels," International Journal of Electrical and Computer Engineering (IJECE), vol.10, no.1, pp. 767-777, 2020.

[21] H. Ibrahim and N. S. P. Kong, "Brightness preserving dynamic histogram equalization for image contrast enhancement," in IEEE Transactions on Consumer Electronics, vol. 53, no. 4, pp. 1752-1758, 2007.

[22] C. H. Ooi, N. S. P. Kong, and H. Ibrahim, "Bi-histogram with a plateau limit for digital image enhancement," in IEEE Transactions on Consumer Electronics, vol. 55, no. 4, pp. 2072-2080, 2009.

[23] C.H. Ooi and N.A.M. Isa, "Quadrants dynamic histogram equalization for contrast enhancement," in IEEE Transactions on Consumer Electronics, vol. 56, no. 4, pp. 2552-2559, 2010

[24] C. H. Ooi and N. A. M. Isa, "Adaptive contrast enhancement methods with brightness preserving," in IEEE Transactions on Consumer Electronics, vol. 56, no. 4, pp. 2543-2551, 2010.

[25] K. Singh and R. Kapoor, "Image enhancement via median-mean based sub-image-clipped histogram equalization," Optik, vol. 125, no. 17, pp. 4646-4651, 2014.

[26] A. S. Parihar, and O. P. Verma, "Contrast enhancement using entropy-based dynamic sub-histogram equalisation," in IET Image Processing, vol. 10, no. 11, pp. 799-808, 2016.

[27] M. Zarie, H. Hajghassem, and A. E. Majd, "Contrast enhancement using triple dynamic clipped histogram equalization based on mean or median," Optik, vol. 175, pp. 126-137, 2018.

[28] M. Zarie, A. Pourmohammad, and H. Hajghassem, "Image contrast enhancement using triple clipped dynamic histogram equalization based on standard deviation," in IET Image Processing, vol. 13, no. 7, pp. 1081-1089, 2019.

[29] R. E. Fischer, B. Tadic-Galeb and P. R. Yoder, "Optical System Design,” Mc Graw-Hill Companies, 2008.

[30] S. B. You, J. W. Kim and S. Kim, "A cmos 16-bit 20msps analog front end for scanner/mfp applications," in IEEE Transactions on Consumer Electronics, vol. 49, no. 3, pp. 647-652, 2003.

[31] A. Rizal, R. Hidayat, and H. Adi Nugroho, "Comparison of Multiscale Entropy Techniques for Lung Sound Classification," Indonesian Journal of Electrical Engineering and Computer Science (IJEECS), vol. 12, no. 3, pp. 948-994, 2018.

[32] K. Singh, R. Kapoor, S. K. Sinha, "Enhancement of low exposure images via recursive histogram equalization algorithms," Optik, vol. 126, no. 20, pp. 2619-2625, 2015.

[33] M. Sudhakara and M. Janaki Meena, "An edge detection mechanism using L*A*B color-based contrast enhancement for underwater images," Indonesian Journal of Electrical Engineering and Computer Science (IJEECS), vol.18, no.1, pp. 41-48, 2020.

[34] G. Zhang, and W. Wang, "Hierarchic Entropy: An Information Theoretic Measure of Evolutionary Robotic Behavioral Diversity," International Journal of Pattern Recognition and Artificial Intelligence, vol. 31, no. 4, pp. 1-13, 2017.

\section{BIOGRAPHIES OF AUTHORS}

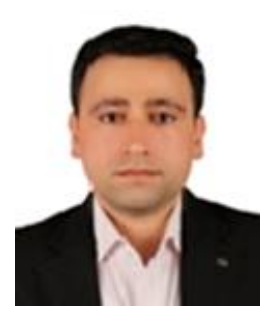

Majid Zarie received his M.S. degree in Electrical-Electronics Engineering from the Imam Hossein Comprehensive University, Tehran, Iran. He received his Ph.D. degree in ElectricalElectronics Engineering from the Malek Ashtar University of Technology, Tehran, Iran. He is at present working as assistant professor in the khatam al-anbia (pbuh) University, Tehran, Iran. His current research interests are optical communications, digital signal processing, and digital image processing. Email:majidzarie@yahoo.com 


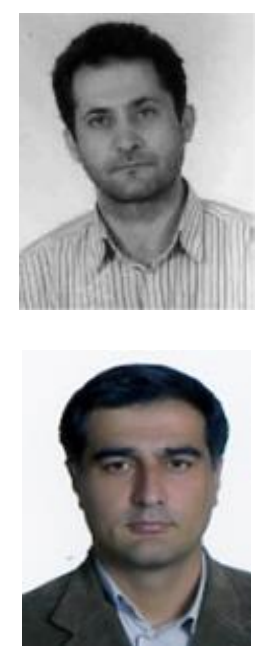

Jafaar Khalilpour received M.S. and Ph.D. degrees from Tarbiat Modares University, Tehran, Iran, in 1998 and 2009, all in electrical engineering. He is at present working as associate professor in the khatam al-anbia (pbuh) University, Tehran, Iran. His research interests include electrical circuits modeling, electromagnetic issues of complex materials, and microwave components. Email: j-khaliepour@ssau.ac.ir

Farhad Sadeghi Almaloo received his M.S. degree in Electrical-Communication Engineering from the Imam Hossein Comprehensive University, Tehran, Iran. He is currently pursuing the Ph.D. degree at the Islamic Azad University, Science and Research Branch, Tehran, Iran. He is at present working as instructor in the khatam al-anbia(pbuh) University, Tehran, Iran His research interests include optical communications, coding theory, digital signal processing, digital image processing, and pattern recognition. Email: farhad.sadeghi@ srbiau.ac.ir 